\title{
Fonoaudiología en cuidados paliativos para adultos y adultos mayores: fundamentos, roles, abordajes y desafíos
}

\author{
Rodrigo Tobar-Fredes ${ }^{\text {a, b }}$, Claudia Olivares-Matus ${ }^{\text {a, } *}$, Sara Tapia-Saavedra ${ }^{\text {a }}$ \\ a Departamento de Fonoaudiología, Universidad de Chile, Chile \\ ${ }^{\text {b } H o s p i t a l ~ d e l ~ T r a b a j a d o r, ~ S a n t i a g o, ~ C h i l e ~}$
}

\begin{abstract}
RESUMEN
La población chilena envejece rápidamente, lo cual se traduce en un incremento en el número de personas que vive con alguna enfermedad crónica que limita su vida, condición que requiere frecuentemente de equipos especializados en cuidados paliativos. En ellos, la inclusión del/la fonoaudiólogo/a a nivel internacional es reciente, aún más en nuestro país. Por lo anterior, son escasas las orientaciones locales que faciliten la activa participación de este profesional bajo un modelo centrado en el cuidado, bienestar y acompañamiento de la persona, su familia y/o personas significativas. En este contexto, es objetivo de esta revisión narrativa el profundizar sobre los fundamentos que soportan la inclusión del/a fonoaudiólogo/a en equipos de cuidados paliativos. Para lo anterior se describen las áreas de acción fonoaudiológica, sus roles y responsabilidades. Al respecto, la información disponible da cuenta de dos grandes campos de acción, vinculados a la comunicación y alimentación, los que deben ser abordados con el propósito de fomentar la participación y autonomía de la persona en la toma de decisiones sobre sus cuidados y el respeto de sus voluntades anticipadas, cuando sea necesario. Bajo este paradigma, los hallazgos son claros en plantear que el/la fonoaudiólogo/a debe alejarse del modelo rehabilitador tradicional, para migrar hacia un grupo de acciones que le permitan abordar -de la mejor manera posible-aquellas condiciones que generan sufrimiento, dolor o molestia a la persona. Dichas acciones son abordadas en esta revisión, con el detalle suficiente para permitir enunciar y proponer seis grandes desafíos para aquello/as profesionales que se desempeñan (o desean hacerlo) en el área.
\end{abstract}

\section{Speech-language pathology in palliative care for adults and older adults: foundations, roles, approaches, and challenges}

\section{ABSTRACT}

The Chilean population is aging rapidly, generating a sustained increase in the number of people living with a chronic disease that limits their life, condition that frequently requires specialized teams in palliative care. In these, the inclusion of the speechlanguage pathologist is recent in most of the countries, even more so in our country. Therefore, there are few local guidelines that facilitate the active participation of this professional under a model focused on the care, well-being and accompaniment of the person, their family and/or significant others. In this context, the objective of this narrative review is to delve into the foundations that support the inclusion of the speech-language pathologist in palliative carea teams. For this, speech-language pathology action areas, roles and responsibilities are described. In this regard, the available information shows two major fields of action. related to communication and swallowing, which must be addressed in orden to promote the participation and autonomy of the person in making decisions about their care, as well as respect for their advance directives, when necessary. Under this paradigm, the findings are clear in stating that the speech-language pathologist should move away from the traditional rehabilitation model, to migrate towards a group of actions that aloe them to address -in the best possible waythose conditions that generate suffering, pain or discomfort to the person. These actions are addressed in this review, with sufficient detail to allow six major challenges to be raised and proposed for those professionals who work (or want to) in the area.

* Autor/a correspondiente: Claudia Olivares-Matus

Email: clolivares@uchile.cl

\section{Palabras clave:} Fonoaudiología; Cuidados paliativos; Cuidados de fin de vida; Comunicación; Alimentación; Deglución

\section{Keywords:} Speech-language pathology; Palliative care; End-of-life care; Communication; Feeding Behavior; Swallowing 


\section{INTRODUCCIÓN}

El envejecimiento de la población, considerado como el aumento en la proporción de personas mayores de 60 años, es actualmente un fenómeno mundial (Albala, 2020). Esta condición se asocia al incremento y acumulación de enfermedades crónicas en la población, entre ellas: demencia, enfermedad pulmonar obstructiva crónica, enfermedad vascular cerebral, cáncer, entre otras (Prince et al., 2015). En Chile, el número absoluto de adultos mayores ha aumentado 6,8 veces entre 1960 y 2017, representando en este último año el $16.2 \%$ de la población del país, con una proyección estimada para el año 2031 de 102 adultos mayores por cada 100 menores de 15 años (Albala, 2020; Instituto Nacional de Estadísticas [INE], 2018). Dichos cambios sociodemográficos ocurren bajo un contexto en que las mejoras e innovaciones en los tratamientos favorecen un incremento en el número de personas que viven con condiciones de salud que limitan su vida, requiriendo de equipos profesionales especializados en cuidados paliativos (Kelly et al., 2016).

Los cuidados paliativos son conceptualizados como la asistencia entregada por un equipo de salud (EdS) con el fin de mejorar la calidad de vida de una persona -su familia y/o personas significativas- que enfrenta(n) los problemas que son propios de una condición de salud potencialmente mortal. Este tipo de asistencia contempla la temprana identificación, evaluación y prescripción de un adecuado manejo de los síntomas y complicaciones -físicos, psicosociales o espirituales-secundarios a la enfermedad precipitante desde realizado el diagnóstico, hasta el final de la vida y durante el transcurso del duelo (Ministerio de Sanidad, Política Social e Igualdad, 2014; National Institute for Clinical Excellence [NICE], 2004; Organización Mundial de la Salud [OMS], 2020). Si bien este tipo de cuidados suele asociarse con el alivio de los síntomas de una persona que está muriendo, estos también pueden ser escogidos por aquellas personas que cursan con enfermedades graves, debilitantes o dolorosas; ya sea por la propia enfermedad o por los efectos de su tratamiento (Pollens, 2012). En este sentido, los cuidados paliativos han de ser aplicados progresivamente a medida que la enfermedad avanza, de acuerdo con las necesidades de la persona enferma -su familia y personas significativas- y no necesariamente solo al final de la vida (Ministerio de Sanidad, Política Social e Igualdad, 2014; OMS, 2020).

Por otra parte, también es frecuente el uso del término cuidados de fin de vida, especialmente para referirse a aquellos servicios entregados durante las últimas semanas de vida, sin embargo, su uso no está necesariamente limitado a tiempos posibles de establecer de manera anticipada. El consenso actual establece su uso para aquellos cuidados -que son parte de los cuidados paliativos- aplicados cuando los síntomas son resultado de una enfermedad que es irreversible y terminal, dejando a la persona con una corta expectativa de vida (Moreira et al., 2020; National Institute for Clinical Excellence [NICE], 2004; Pollens, 2012). De este modo, los cuidados de fin de vida obligan al EdS a replantearse los objetivos terapéuticos, debiendo individualizar la decisión terapéutica siempre respetando los deseos y decisiones de la persona; al tiempo que se omiten y suspenden todos aquellos tratamientos que no se dirigen exclusivamente a producir alivio (Aguirre Bravo \& Sampallo Pedroza, 2015).

$\mathrm{Si}$ bien las anteriores definiciones resultan una adecuada operacionalización para ambos tipos de cuidados, es importante reforzar que estos no deben ser simplificados a una serie de procedimientos o a un tipo de abordaje terapéutico. Por el contrario, los cuidados paliativos y de fin de vida deben ser considerados como una política pública que integra a todo el sistema de salud -y cada uno de sus niveles de complejidadpermitiendo el desempeño de un equipo interdisciplinario capaz de entregar un cuidado comprehensivo, integrado, humanizado y de alta calidad técnica (Moreira et al., 2020). Por ello, quienes forman parte de estos equipos, deben lograr comprender y empatizar con las necesidades de la persona en cuidados paliativos (PeCP), su familia $\mathrm{y} / \mathrm{o}$ personas significativas; transformándose en un soporte durante la toma de decisiones hacia el final de la vida, brindándoles dignidad, respeto y constante apoyo en el afrontar la enfermedad y aceptar la muerte como una etapa natural de la vida (Moreira et al., 2020). Dicho soporte profesional ha de estar regido siempre por el principio ético de autonomía, permitiendo resguardar tanto la integridad humana como el respeto de las creencias y deseos que posee la PeCP respecto al final de su vida (Burlá \& Py, 2014; Moreira et al., 2020).

En este contexto, es reconocido que las personas que se encuentran al final de su vida viven importantes cambios en su comunicación y alimentación, los que se encuentran presentes incluso en aquellas personas que cursan con condiciones de salud que usualmente no se asocian a este tipo de alteraciones (Kelly et al., 2016). Dicha situación ha derivado en la progresiva inclusión del/la fonoaudiólogo/a en EdS que ofrecen sus servicios bajo el marco de atención de los cuidados paliativos (Aguirre Bravo \& Sampallo Pedroza, 2015; Kelly et al., 2016; Prelock et al., 2017). Ello, convoca a estos/as profesionales a desarrollar nuevas competencias y, subsecuentemente, a cambiar el foco de su intervención, migrando desde un abordaje rehabilitador a uno con orientación hacia la prevención y el alivio de síntomas (Kelly et al., 2016). Así, el tradicional rol del/la fonoaudiólogo/a es 
puesto en tensión, siendo necesaria una profunda revisión sobre su quehacer como parte de un equipo interdisciplinario de cuidados paliativos, el cual debe estar en congruencia con la información disponible en la literatura científica y las particularidades del sistema de salud de cada país. De este modo, son objetivos de esta revisión: (1) caracterizar el rol del/la fonoaudiólogo/a que se desempeña en cuidados paliativos, documentando los fundamentos de su quehacer, (2) sintetizar la evidencia del trabajo fonoaudiológico al interior de equipos de cuidados paliativos, a modo de sugerencias para el abordaje de la deglución y comunicación, y (3) establecer aquellos desafíos que surgen del quehacer fonoaudiológico en esta área emergente para la disciplina.

\section{ESTRATEGIA DE BÚSQUEDA}

Para alcanzar el objetivo planteado se realizó una revisión de la literatura centrada en la práctica fonoaudiológica en cuidados paliativos de personas adultas. Se utilizó la técnica descrita por Arksey \& O'Malley (2005) para un scoping review, siendo consideradas las bases de datos PubMed, Scopus y Scielo. Los términos de búsqueda fueron: "palliative care" y "end of life". Estos, combinados de manera separada, a través del operador boleano "AND", con los términos truncados "speech therap"” y "speech patholog*". Fueron excluidos todos aquellos artículos publicados antes de 1998, sin resumen disponible o correspondientes a presentaciones en conferencias. Además, se realizó una revisión amplia de la literatura gris, incorporando documentos vinculados a la temática publicados por organismos de salud oficiales (ej.: National Institute for Health and Care Excellence [NICE], del Reino Unido).

A continuación, se presentan los resultados más relevantes, los cuales permiten responder al objetivo planteado para esta revisión. En primer lugar, se establecen los aspectos generales que enmarcan el desempeño profesional de fonoaudiólogos y fonoaudiólogas en cuidados paliativos. Luego, se sintetiza la información disponible en la literatura existente a la fecha sobre los roles que debe cumplir el/la profesional fonoaudiólogo/a que se desempeña en esta área. Posteriormente, se entregan una serie de sugerencias para el abordaje fonoaudiológico de la deglución y comunicación en cuidados paliativos basadas en la evidencia actual. Por último, tras un análisis pragmático de la información revisada, se plantea una serie de desafíos que emergen de la inclusión del/a fonoaudiólogo/a en cuidados paliativos bajo las características actuales del sistema de salud chileno.

\section{CONTEXTO DE DESEMPEÑO PROFESIONAL}

Los/as fonoaudiólogo/as que se desempeñan en cuidados paliativos deben desarrollar habilidades que les permitan cumplir de manera satisfactoria nuevos roles, integrando sus servicios y conocimientos con los de otros/as integrantes del EdS, en correspondencia con las necesidades de la persona que se acerca al final de su vida, su familia y/o personas significativas (Pollens, 2012). Al respecto, en concordancia con la evidencia disponible a la fecha, es posible establecer que el ejercicio profesional de fonoaudiólogo/as en cuidados paliativos ha de estar inscrito bajo una serie de condiciones que son propias a este tipo de atención en salud. Entre estos, y para fines de esta revisión, se abordarán aquellos que permiten establecer el contexto general en el cual ha de participar y colaborar el/la profesional de la comunicación y alimentación oral, a decir: (a) valores y principios, y (b) conformación del equipo de salud.

\section{Valores y principios de los cuidados paliativos}

En consideración de la complejidad que implica para el EdS abordar decisiones a propósito de los cuidados paliativos incluidos los cuidados de fin de vida-, la literatura describe una serie de lineamientos sobre los cuales todo/a profesional de la salud que presta servicios en el área, incluidos fonoaudiólogo/as, debe adscribir resguardando su estricto cumplimiento. Al respecto, y de acuerdo a lo planteado por el Ministerio de Sanidad, Política Social e Igualdad (2014), a continuación se sintetizan aquellos valores que buscan resguardar un trato respetuoso hacia la PeCP y sus personas significativas, como también los principios que han de enmarcar el actuar de todo el EdS (ver Tabla $1)$. 
Tabla 1. Valores y Principios de los Cuidados Paliativos, generado a partir del documento "Estrategias en Cuidados Paliativos del Sistema Nacional de Salud" (Ministerio de Sanidad, Política Social e Igualdad, 2014).

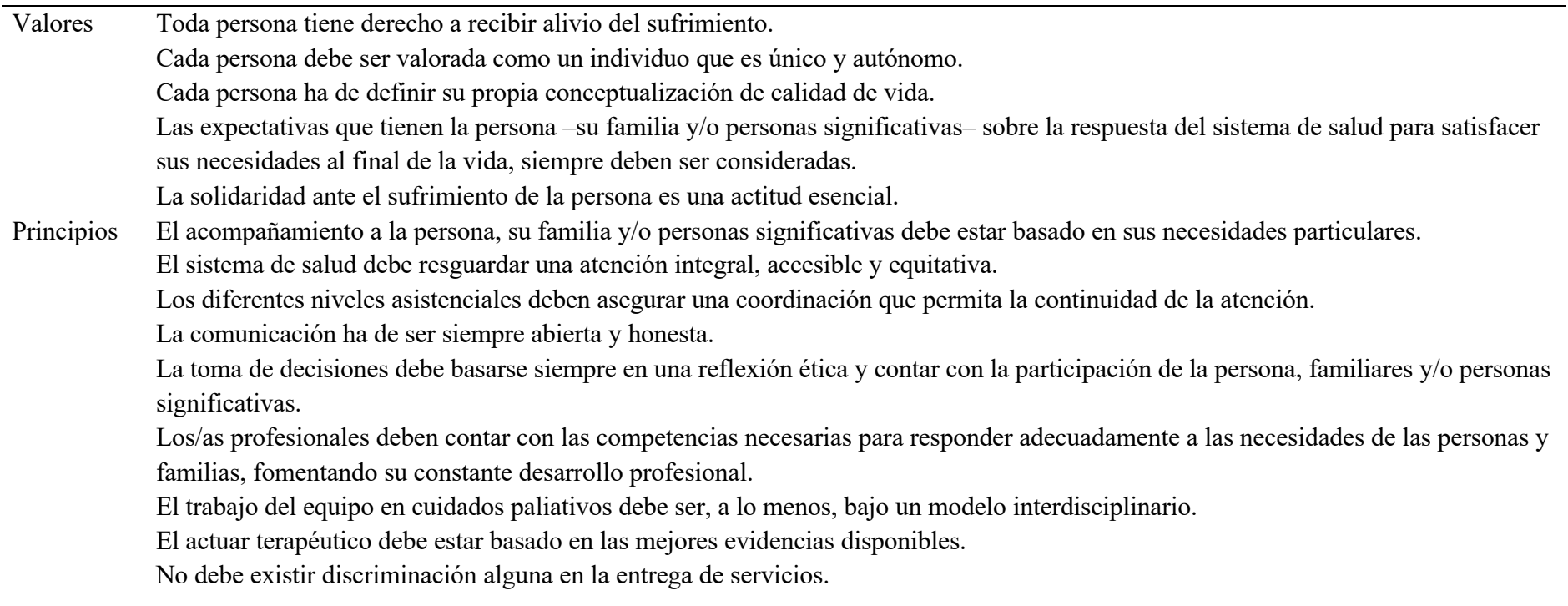

\section{Conformación del equipo de cuidados paliativos}

En cuanto a su conformación, hasta hace no muchos años, los equipos de cuidados paliativos correspondían a pequeños grupos de profesionales compuestos principalmente por médicos/as y enfermeros/as. Actualmente, se observa un paulatino cambio en la conformación de estos equipos, mostrando la progresiva incorporación de otro/as profesionales, tales como kinesiólogo/as, terapeutas ocupacionales, psicólogos/as, farmacéutico/as, trabajadores sociales, y fonoaudiólogo/as. Al respecto, la evidencia demuestra que esta incorporación conlleva positivos beneficios para la entrega de servicios en cuidados paliativos (Pollens, 2012). Dicha situación ocurre bajo la recomendación actual que insta a utilizar el modelo biopsicosocial como marco de acción en cuidados paliativos, posicionando a la PeCP, su familia y/o personas significativas como miembros nucleares del equipo, favoreciendo su activa participación en la toma de decisiones.

Bajo este contexto se inserta la participación de fonoaudiólogo/as en cuidados paliativos, quienes - bajo el modelo biopsicosocialhan de propiciar, junto al resto de los integrantes del EdS, formatos de trabajo idealmente interdisciplinarios, al tiempo que aportan en lo específico mediante el cumplimiento de una serie de roles que emergen en la literatura actual como parte de sus responsabilidades. Dichos roles son presentados a continuación.

\section{ROLES DEL FONOAUDIÓLOGO Y LA FONAUDIÓLOGA EN CUIDADOS PALIATIVOS}

A nivel internacional, las primeras iniciativas que incluyen a fonoaudiólogos/as en equipos de cuidados paliativos surgen a propósito de su participación en el manejo de personas con cáncer (Eckman \& Roe, 2005). Desde entonces, su integración a equipos especialistas en cuidados paliativos para personas con enfermedades no oncológicas se mantiene en desarrollo. Dicho proceso se ha acompañado de un progresivo cambio en la percepción de PeCP, familias y otro/as profesionales de la salud, sobre la necesidad de contar con un/a profesional que provea soporte en las dificultades de la comunicación y alimentación experimentadas por la PeCP; siendo actualmente reconocida su labor por diversos documentos y guías clínicas internacionales (Eckman \& Roe, 2005; NICE, 2004; Prelock et al., 2017; Royal College of Physicians and British Society of Gastroenterology, 2010). Pese a lo anterior, el valor que se le otorga a aquello/as fonoaudiólogo/as especialistas en cuidados paliativos aún no es del todo reconocido por fonoaudiólogo/as generales, principalmente producto del escaso conocimiento que poseen estos últimos sobre las particularidades del abordaje fonoaudiológico realizado al interior de $\mathrm{EdS}$ en cuidados paliativos (Kelly et al., 2016; Toner \& Shadden, 2012; Vescovich, 2015).

En Chile, sumado a lo ya descrito, una de las principales limitaciones que ha dificultado el posicionamiento de la fonoaudiología en cuidados paliativos es la escasa investigación 
existente respecto a la práctica y desafíos que esto/as profesionales han de enfrentar durante su desempeño, sumado a la falta de protocolos específicos para su proceder en nuestro país.

Tomando en consideración lo expuesto, resulta recomendable rescatar aquellos aspectos que han demostrado ser relevantes para aquellos/as fonoaudiólogo/as que forman parte de equipos de cuidados paliativos en países con mayor experiencia en el área. $\mathrm{Al}$ respecto, el estudio realizado por Chahda et al. (2020) entregó un detallado análisis de los aspectos centrales de la intervención fonoaudiológica, estableciendo siete roles cardinales para su desempeño en cuidados paliativos (ver Tabla 2).

Tabla 2. Roles del/la fonoaudiólogo/a en cuidados paliativos, traducido y adaptado por los autores a partir de Chahda et al. (2020).

\begin{tabular}{|c|c|}
\hline Tipo de participación (rol) & Definición \\
\hline Intercesión & Interceder para que la PeCP reciba información respecto a su estado deglutorio y comunicativo. \\
\hline Identificación & $\begin{array}{l}\text { Identificar la necesidad de incorporar aportes fonoaudiológicos en el manejo clínico de la } \mathrm{PeCP}-\mathrm{o} \text { bien, un cambio en } \\
\text { el abordaje- que permitan promover el confort y el respeto de las preferencias de la persona, en contraposición a } \\
\text { aquellos objetivos estrictamente rehabilitadores. }\end{array}$ \\
\hline Evaluación & $\begin{array}{l}\text { Evaluar la deglución, comunicación y habilidades cognitivas de la PeCP, con el propósito de monitorear los cambios } \\
\text { que ocurren a lo largo del continuo paliativo. }\end{array}$ \\
\hline Manejo & $\begin{array}{l}\text { Crear un plan de manejo a implementar -y modificar- en concordancia con los cambios que tengan las necesidades y } \\
\text { habilidades de la PeCP, asegurando un cuidado con foco en la persona y su confort. }\end{array}$ \\
\hline Soporte & $\begin{array}{l}\text { Apoyar y abogar para que la PcEP reciba una atención que respete sus preferencias personales en deglución y } \\
\text { comunicación, bajo la guía de un/a fonoaudiólogo/a experimentado/a. }\end{array}$ \\
\hline Asesoría y consultoría & $\begin{array}{l}\text { Brindar asesoría educacional y apoyo emocional a la PeCP y su familia, favoreciendo su adaptación a los cambios } \\
\text { deglutorios y comunicativos vinculados a la enfermedad y su progresión. }\end{array}$ \\
\hline \multirow[t]{2}{*}{ Educación } & $\begin{array}{l}\text { Educar a la PeCP, su familia y/o personas significativas, empoderándolos para involucrarse activamente en el manejo } \\
\text { de la deglución y comunicación. }\end{array}$ \\
\hline & $\begin{array}{l}\text { Educar al equipo interdisciplinario sobre el manejo de la deglución y comunicación, el rol del/la fonoaudiólogo/a, y } \\
\text { cuándo derivar a este/a profesional. }\end{array}$ \\
\hline
\end{tabular}

La consolidación del/la fonoaudiólogo/a en cada uno de estos roles cardinales requiere necesariamente de una modificación en sus modelos de intervención, lo cual expande su campo de acción más allá de las tradicionales intervenciones directas en la PeCP, su familia y/o personas significativas. Al respecto, Holland \& Nelson (2007) argumenta que el/la profesional debe asumir la labor de consejero/a o asesor/a del equipo interdisciplinario; otorgando soporte y orientaciones para el adecuado manejo de las alteraciones de la comunicación y alimentación. Según diversos autores, lo anterior da cuenta de la necesidad de que el/la fonoaudiólogo/a desarrolle su capacidad para ajustar sus procedimientos de evaluación y manejo convencionales a las necesidades de la PeCP, su familia y/o personas significativas; facilitando así su adecuada inserción en un equipo de cuidados paliativos (Chahda et al., 2017; Kelly et al., 2016; Prelock et al., 2017).

En adición a lo ya expuesto, la literatura es enfática en resaltar que cada uno de los roles previamente descritos deben ser desenvueltos por el/la fonoaudiólogo/a de manera flexible, siempre en concordancia con las características particulares de los diferentes escenarios clínicos posibles, incluidos: hospitales, establecimientos de larga estadía, hospitalización domiciliaria u otros (Toner \& Shadden, 2012). En cada uno de estos, el/la fonoaudiólogo/a debe dar cuenta de dos grandes áreas de ejercicio profesional: deglución y comunicación (Chahda et al., 2017, 2020; Kelly et al., 2016; Pollens, 2012).

Es importante señalar que, aunque para ambas áreas de desempeño aún existe una incipiente producción científica, los hallazgos descritos a la fecha son suficientes para entregar una serie de sugerencias que buscan resguardar un adecuado abordaje fonoaudiológico en cuidados paliativos, los cuales son expuestos a continuación.

\section{SUGERENCIAS PARA EL ABORDAJE DE LA DEGLUCIÓN Y ALIMENTACIÓN EN CUIDADOS PALIATIVOS}

La deglución corresponde a una habilidad prioritaria en los cuidados de toda persona, estando a la base de funciones tan 
relevantes para el ser humano como son la hidratación, nutrición, medicación y manejo de secreciones. Lamentablemente, en el contexto de los cuidados paliativos, se ha demostrado que las condiciones de salud que sobrellevan las PeCP frecuentemente derivan en una alteración de la función deglutoria, ya sea por los efectos propios de la enfermedad o por aquellos derivados de su tratamiento (Langmore et al., 2009), interfiriendo significativamente en el bienestar y calidad de vida de la persona afectada (Kelly et al., 2016). Dicha situación supone un complejo desafío clínico y ético, en el cual participa no solo el EdS, sino también la familia, personas significativas, cuidadores y la propia PeCP. A continuación, se sintetiza la evidencia disponible respecto a las consideraciones que han guiar la práctica clínica y la toma de decisiones de lo/as fonoaudiólogo/as en el abordaje de la deglución y alimentación de PeCP. Entre ellas, la caracterización clínica de los tipos de usuarios y el uso de sistemas alternativos para la alimentación e hidratación, entre otros.

\section{Tipos de usuarios y consideraciones generales para el abordaje}

Los cambios que ocurren en la deglución y alimentación durante el continuo de los cuidados paliativos permiten la caracterización de $\mathrm{PeCP}$ con diferente expresión clínica. Al respecto, y tomando como base lo descrito previamente por Evans \& Best (2015), proponemos a continuación dos grandes grupos:

a. Personas en cuidados paliativos con disfagia, quienes a consecuencia de su condición/etiología de base, cursan con una alteración deglutoria que disminuye la capacidad para proteger su vía aérea, presentando una alta probabilidad o riesgo de aspirar y/o ahogarse.

b. Personas en cuidados paliativos con una alteración en su alimentación, las cuales presentan una capacidad deglutoria funcional, pero que al acercarse al final de sus vidas pierden: la habilidad para reconocer el hambre o la sed, el deseo de ingerir alimentos y/o líquidos, la capacidad para reconocer el contenido oral, o bien, una conducta para la alimentación alterada producto de cambios cognitivos y/o emocionales.

Este contexto, la evidencia instiga a lo/as fonoaudiólogo/as que son parte de equipos de cuidados paliativos a movilizar sus conocimientos y competencias para lograr una detallada descripción de la condición particular de cada PeCP, colocando especial énfasis en la determinación de cuán segura es la deglución (Kelly et al., 2016; Langmore et al., 2009; Royal College of Physicians and British Society of Gastroenterology, 2010; Toner \& Shadden, 2012); y cuán eficaz y confortable resulta la ingesta, en caso de ser posible. Posteriormente, dicha información debe ser compartida con cada uno de los integrantes del EdS, familia, personas significativas y PeCP para la toma conjunta de directrices (recomendaciones, pautas y decisiones) específicas en el área, siempre con una orientación hacia la mejor calidad de vida posible. Al respecto, la literatura es clara en reforzar que todas las directrices que resulten del proceso anterior deben estar basadas en un profundo análisis y valoración de los beneficios (y riesgos) que pueden derivar del mantener (o no) la ingesta por vía oral, o bien, el uso de una vía artificial; resoluciones en las cuales el/la fonoaudiólogo/a ha de participar activamente en sus roles de educador/a, asesor/a, consultor/a e interventor/a.

\section{Consideraciones para la toma de decisiones en deglución y alimentación}

Respecto a las prácticas recomendadas en la literatura para el proceso de evaluación, es preponderante la evaluación clínica, la cual permite describir de manera más ecológica el rendimiento funcional de la PeCP en su deglución y alimentación; así como el contexto en el que ocurren, sus barreras y facilitadores. La evidencia muestra que el uso de la evaluación instrumental puede ser útil, siempre y cuando los beneficios que se obtengan de sus resultados permitan incrementar el bienestar de la persona, mediante la optimización y/o maximización las capacidades de la persona para comer o beber (Kelly et al., 2016).

Tal como se ha mencionado previamente, es importante reforzar que la literatura desliga al abordaje fonoaudiológico de la deglución y alimentación en cuidados paliativos de los principios del modelo clásico o rehabilitador. Bajo este contexto, la evidencia permite establecer otro tipo de objetivos, siendo más adecuados para los cuidados paliativos: la reducción de la aspiración, el manejo de las variables que pueden desencadenar una neumonía (ej.: higiene oral), y el reducir la malnutrición y/o deshidratación (Kelly et al., 2016). Dichos objetivos resultan particularmente relevantes en aquellas $\mathrm{PeCP}$ donde no es posible eliminar la aspiración, situaciones en las que se plantea como perentorio que el/la profesional migre desde un abordaje centrado en la "minimización de los riesgos", hacia uno orientado al "manejo de los riesgos" (Eckman \& Roe, 2005). Lo anterior, es propuesto bajo un modelo que contempla la evaluación constante durante todo el curso de la enfermedad, permitiendo realizar los ajustes necesarios al plan de manejo en concordancia con los cambios en la condición de la persona.

En lo práctico, los antecedentes existentes a la fecha establecen que el/la fonoaudiólogo/a debe asistir en el desarrollo de estrategias para maximizar la habilidad de la PeCP para disfrutar 
el comer y/o beber de la manera más segura y confortable por el mayor tiempo que sea posible (Chahda et al., 2017; Pollens, 2012). Durante este trayecto, diversas son las variables descritas para su consideración en el logro de un adecuado equilibrio entre riesgos y beneficios durante el desarrollo o implementación de un plan de intervención, especialmente en aquellas $\mathrm{PeCP}$ con alto riesgo de aspiración. Al respecto, una guía que puede resultar útil para lo/as clínico/as es el estudio realizado por Berkman et al.
(2019), a propósito del manejo de la deglución en personas con demencia avanzada. De este estudio destacan cinco temas centrales (ver Tabla 3), que se recomiendan incorporar -con las adecuaciones necesarias para el contexto nacional- durante el proceso de toma de decisiones del/a fonoaudiólogo/a junto al equipo interdisciplinario, la $\mathrm{PeCP}$, su familia y personas significativas.

Tabla 3. Temas centrales que ha de considerar el/la fonoaudiólogo/a para el manejo de la deglución y alimentación de personas en cuidados paliativos, traducido y adaptado por los autores a partir de Berkman et al. (2019).

\begin{tabular}{|c|c|}
\hline Tema & Descriptor \\
\hline Deseos de la persona & $\begin{array}{l}\text { La recomendación de mantener la ingesta oral, o bien, considerar el uso de una vía de alimentación artificial ha de estar } \\
\text { soportada en las voluntades anticipadas de la PeCP. Idealmente, estas deben ser comunicadas a la familia de manera } \\
\text { previa, cumpliendo el/la fonoaudiólogo/a un importante rol en facilitar conversaciones, entre ambas partes, abordando el } \\
\text { tema con la suficiente antelación. Para aquellas situaciones en que no se abordó el tema, se ha de instar a la familia a guiar } \\
\text { su toma de decisiones en base a lo que PeCP desearía, reconociendo con ello que son quienes mejor le conocen. }\end{array}$ \\
\hline Calidad de vida & $\begin{array}{l}\text { La mantención de la ingesta oral ha sido recomendada para el confort y placer, particularmente en aquellas personas que } \\
\text { están al final de sus vidas. Este tipo de ingesta debe ser considerada particularmente para personas que mantienen la } \\
\text { habilidad para comer/beber y demuestran interés o placer por la ingesta; siempre bajo un argumento que priorice la calidad } \\
\text { de vida. }\end{array}$ \\
\hline $\begin{array}{l}\text { Minimización del } \\
\text { riesgo de neumonía }\end{array}$ & $\begin{array}{l}\text { La ingesta oral puede ser recomendada idealmente bajo un contexto clínico en el cual es posible mitigar el riesgo de } \\
\text { aspiración. Sin embargo, esto no siempre es factible, siendo el objetivo mayor de la intervención fonoaudiológica el } \\
\text { minimizar el riesgo de neumonía. Esto incluye, a lo menos, el uso de adecuaciones compensatorias, maximización de la } \\
\text { higiene oral y modificaciones en la dieta. }\end{array}$ \\
\hline $\begin{array}{l}\text { Tomas de decisiones } \\
\text { informadas }\end{array}$ & $\begin{array}{l}\text { En la mayoría de las ocasiones, es la familia (o persona responsable de la PeCP) quien toma la decisión final sobre } \\
\text { mantener o no la ingesta por vía oral. Para ello, el/la fonoaudiólogo/a -junto al equipo de salud- debe informar sobre los } \\
\text { beneficios y riesgos de mantener una ingesta oral; permitiendo una toma de decisiones que se soporte en toda la } \\
\text { información disponible sobre la condición particular de la PeCP. }\end{array}$ \\
\hline $\begin{array}{l}\text { Evidencia sobre el uso } \\
\text { de alimentación } \\
\text { artificial }\end{array}$ & $\begin{array}{l}\text { La actual evidencia no apoya el uso de sistemas de alimentación artificial en personas que entran en una fase de "cuidados } \\
\text { de fin de vida". El uso de sondas u ostomías en esta población no disminuye los riesgos de aspiración o desnutrición, } \\
\text { tampoco incrementan la calidad de vida y, por el contrario, conlleva otros riesgos asociados. }\end{array}$ \\
\hline
\end{tabular}

\section{Uso de vías alternativas para la nutrición e hidratación}

Es relevante señalar que la evidencia disponible a la fecha no recomienda el uso de vías alternativas que facilitan la nutrición e hidratación artificial. Al respecto, los antecedentes actuales describen que la indicación de sondas u ostomías, como solución final para la disfagia en $\mathrm{PeCP}$, puede significar consecuencias adversas de difícil manejo. En personas que aún mantienen cierto grado de participación, el uso de sondas se ha vinculado a situaciones como la exclusión de la $\mathrm{PeCP}$ de las comidas, incrementando su aislamiento social, seguido por un aumento del tiempo en cama, favoreciendo el fracaso de todo intento por optimizar su condición, y acelerando la muerte de algunas personas. Ello, cuando la intención original de su indicación era mejorar su estado de salud (Langmore et al., 2009). Respecto a las ostomías, la evidencia actual reporta que su uso en quienes se acercan al final de su vida -especialmente aquellas personas mayores con deterioro cognitivo- tampoco demuestra mejora alguna en su pronóstico o calidad de vida (Gillick, 2000; Mamun \& Lim, 2005; Naruishi \& Nishikawa, 2018).

A pesar de lo anterior, la indicación de vías de alimentación alternativa -especialmente ostomía- sigue siendo frecuente en la práctica clínica chilena de los cuidados paliativos, bajo el argumento de sus supuestos beneficios para prevenir la neumonía aspirativa, mejorar los parámetros nutricionales y evitar lesiones por presión (Portales, 2015). Dichos argumentos son revisados a continuación en perspectiva de los resultados de la revisión realizada: 
Vías de alimentación alternativa como medida para prevenir la aspiración

La literatura científica actual, cada vez más numerosa, ha demostrado que el uso de sondas y ostomías no logra prevenir la aspiración en aquellas personas a las cuales se les ha prescripto, incluidas aquellas en cuidados paliativos o de fin de vida. Así mismo, su indicación ha demostrado ser insuficiente para disminuir la prevalencia de neumonía. Muy por el contrario, y en específico para $\mathrm{PeCP}$, su uso parece estar asociado a un incremento en la incidencia de neumonía, en comparación a aquellas personas que mantienen algún tipo de ingesta oral (Cintra et al., 2014).

En particular, el uso de sondas en personas con demencia avanzada ha demostrado generar un incremento en la incidencia de neumonía aspirativa, la cual incluso duplica la de personas que, bajo la misma condición de salud, no tienen indicación de uso de sonda (Alvarez-Fernández et al., 2005; Arinzon et al., 2008; Cintra et al., 2014; Ijaopo \& Ijaopo, 2019). Al respecto, se describen dos posibles principales causas (Gomes et al., 2003): (1) la administración de alimentación a través de una sonda aumenta el $\mathrm{pH}$ gástrico, derivando en una colonización bacteriana de las secreciones gástricas, las cuales pueden migrar utilizando la sonda como medio para subir desde el estómago -a través del esófago- y colonizar la faringe, y (2) la restricción de la ingesta oral, es responsable de una baja en la secreción salival, la cual deriva en la colonización de bacterias gram negativo en la cavidad orofaríngea y la sonda enteral, las cuales posteriormente ingresan junto a las secreciones hacia la vía respiratoria inferior.

El uso de ostomías ha demostrado un riesgo similar al de las sondas enterales para la generación de neumonía aspirativa; observándose solo un probable decremento mediante el uso de yeyunostomía, pero en poblaciones con condiciones muy particulares (Luk \& Chen, 2014). Una de las causas más frecuente de neumonía en personas con ostomía corresponde a la hiperalimentación, la cual puede generar que la fórmula refluya hacia el esófago y faringe generando aspiración (Levy et al., 2004). Del mismo modo, la falta de higiene oral es también una causa relevante, toda vez que se ha demostrado que los cuidadores de $\mathrm{PeCP}$ tienden a descuidar los cuidados orales cuando se usa una alimentación enteral (Ijaopo \& Ijaopo, 2019; Teramoto et al., 2006).

Tampoco se han encontrado diferencias por la forma en que se ingresan los alimentos al sistema de alimentación artificial. El uso de bombas de infusión continuas no ha demostrado ser mejor que la alimentación intermitente para la prevención de neumonía (Luk \& Chen, 2014).

\section{Vías de alimentación alternativa como medida para una mejora en la condición física y/o nutricional}

La alimentación artificial no siempre logra mejoras en el funcionamiento físico, el aumento de peso, el estado nutricional o la regeneración de heridas. De hecho, se ha demostrado que su uso no tiene efectos sobre la malnutrición de personas con enfermedades crónicas, ni ayuda en la recuperación en aquellas que tienen lesiones por presión $\mathrm{y}$, por el contrario, se les ha asociado a un mayor riesgo de muerte y peor calidad de vida (Alvarez-Fernández et al., 2005; Arinzon et al., 2008; Cintra et al., 2014; Finucane et al., 1999; Ijaopo \& Ijaopo, 2019; Levy et al., 2004) (Alvarez-Fernández et al., 2005; Arinzon et al., 2008; Cintra et al., 2014; Finucane, 2000; Ijaopo \& Ijaopo, 2019; Levy et al., 2004). Sin embargo, se han demostrado mejoras en la calidad de vida de quienes ejercen el rol de cuidadore/as; disminuyendo su sobrecarga y estrés asociado a la alimentación de la PeCP (Nakanishi \& Hattori, 2014), siendo este un dato relevante para la evaluación de la pertinencia de indicar/mantener (o no) un sistema de estas características, dado que muchas veces solo se puede acceder a la opinión de los cuidadores, la cual no necesariamente da cuenta de las necesidades/deseos de la PeCP.

En el caso de aquellas personas que inician con cuidados de final de vida, existen algunas consideraciones adicionales. Así, se establece que en etapas más cercanas al final de la vida -en el contexto de una persona con cáncer, por ejemplo- un indicador pronóstico de que la expectativa de vida ha disminuido a semanas o días es la pérdida de interés por los alimentos o líquidos (NICE, 2004; Royal College of Physicians and British Society of Gastroenterology, 2010), condición que se replica para otras condiciones terminales (Carter, 2020; Rochford, 2021). En este contexto, la aplicación de estrategias que favorecen un buen cuidado e higiene oral resultan más apropiadas que cualquier otra intervención orientada a intentar algún tipo de ingesta oral. Esta condición supone, además, evaluar si continúa siendo apropiada la nutrición e hidratación por una vía alternativa, si esta ya fue implementada (Carter, 2020; Rochford, 2021; Royal College of Physicians and British Society of Gastroenterology, 2010). Al respecto, el descontinuar fluidos por vía parenteral debe ser considerado, tomando en cuenta que en estados avanzados de la enfermedad su mantención favorece la exacerbación del edema pulmonar y periférico; incrementando la producción de secreciones, las cuales una PeCP con bajo nivel de conciencia no es capaz de manejar (Rochford, 2021; Royal College of Physicians and British Society of Gastroenterology, 2010). 
En línea con lo anterior, las aún escasas guías clínicas existentes a la fecha (e.j., Royal College of Physicians and British Society of Gastroenterology (2010)) establecen que todo sistema de nutrición e hidratación artificial resulta inapropiado para situaciones de salud en las cuales:

(1) El riesgo del procedimiento es mayor a los beneficios esperados,

(2) La PeCP rechaza su implementación, o lo ha hecho saber al equipo y/o familia como una voluntad anticipada,

(3) $\mathrm{La} \mathrm{PeCP}$ tiene un mal pronóstico y escasas probabilidades de sobrevivir, $\mathrm{y}$

(4) La PeCP cursa con una demencia avanzada, situaciones en las cuales se ha demostrado muy escasa evidencia que reconozca su aporte en la mejora de la calidad de vida o en el prolongar la sobrevida.

Finalmente, si bien la evidencia expuesta apunta a evitar el uso de vías de alimentación artificial en cuidados paliativos, especialmente durante el período de cuidados de fin de vida, resulta trascendental que cada equipo interdisciplinario evalúe caso a caso la decisión -junto a la familia y/o personas significativas- evitando utilizar los antecedentes mencionados como argumentos a ciegas y sin el adecuado correlato clínico; reconociendo además la amplia variabilidad que existe entre las personas que son sujeto/as de recibir cuidados paliativos.

\section{Lineamientos generales para la práctica en deglución y alimentación}

A partir de lo descrito en este apartado, se desprende que es función del/la fonoaudiólogo/a determinar constantemente dando seguimiento a los cambios producto de la enfermedad de base- la funcionalidad deglutoria, la capacidad de alimentación, la higiene oral y el rendimiento cognitivo-conductual de la PeCP. Dichos datos deben ser analizados en conjunto a todos aquellos otros factores -contextuales y ambientales- que pudiesen favorecer (o no) cualquier tipo de ingesta. Para ello, el/la profesional debe considerar todas las estrategias -especialmente compensatorias- que son parte de su arsenal clínico, que le permitan mantener la ingesta por vía oral durante el mayor tiempo posible y, por sobretodo, la preservación de la respuesta motora orofaríngea para la deglución de sus secreciones.

En el caso de aquellas PeCP que presentan una disfagia severa, o ingresan a un programa de cuidados de fin de vida, se recomienda considerar abordajes orientados a la ingesta por confort. Al respecto, la evidencia establece que estos deben ser incorporados tan pronto como sea posible, siempre y cuando la persona así lo solicite o requiera a fin de mejorar su calidad de vida. Para ello, la recomendación a la fecha es que su uso debe estar siempre bajo un enfoque que minimice los riesgos de generar neumonía aspirativa (por sobre uno que minimice la aspiración), utilizando para ello cantidades mínimas de alimentos o líquidos, que permitan a la persona disfrutar o sentir placer a través de diferentes sabores, texturas y/o aromas (Fong et al., 2020; Palecek et al., 2010). A lo anterior, la literatura también considera la humectación de la cavidad oral con líquidos como una estrategia relevante para incrementar el confort, permitiendo disminuir la sensación de sed o el malestar de una cavidad oral seca (Aguirre Bravo \& Sampallo Pedroza, 2015; Palecek et al., 2010). En su conjunto, este grupo de acciones ha sido planteado como una estrategia relevante en la totalidad de las guías más recientes, fomentándose su incorporación en el manejo de toda PeCP sin ingesta por vía oral; independientemente de si cuentan con una vía alternativa de nutrición e hidratación.

\section{SUGERENCIAS PARA EL ABORDAJE DE LA COMUNICACIÓN EN CUIDADOS PALIATIVOS}

Como resultado del progreso de la enfermedad, las capacidades comunicativas de la $\mathrm{PeCP}$ disminuyen progresivamente, siendo necesaria una serie de estrategias orientadas a incrementar su eficacia comunicativa (Pollens, 2012). La evidencia establece que dichas estrategias deben ser guiadas por un/a fonoaudiólogo/a adecuadamente entrenado/a en cuidados paliativos, facilitando la prosecución de los objetivos subyacentes a la intervención comunicativa.

\section{Objetivos de la intervención comunicativa}

Por lo general, las habilidades comunicativas de la $\mathrm{PeCP}$ afectan la interacción con sus interlocutores, transformándola en un acto que se vuelve poco predecible debido a los efectos de las fluctuaciones en el nivel de alerta, fatiga, dolor, respuesta a la medicación y la propia progresión de la enfermedad de base (Chahda et al., 2017; Pollens, 2012). En este contexto, diversos autores plantean que el/la clínico/a ha de determinar las capacidades cognitivo-comunicativas de la $\mathrm{PeCP}$, información que posteriormente debe ser ofrecida al equipo interdisciplinario, apoyándolo en la determinación de la capacidad que posee la persona para tomar decisiones y manifestar sus deseos o necesidades (Kelly et al., 2016). Dicha información debe ser considerada en las resoluciones del equipo y la familia, fomentando la participación de la $\mathrm{PeCP}$ durante el mayor tiempo que sea posible. Paralelamente, la intervención fonoaudiológica debe maximizar la efectividad comunicativa, como una estrategia 
orientada esencialmente a disminuir el sufrimiento de la PeCP (Goldberg, 2007; Toner \& Shadden, 2012).

Bajo este contexto, la literatura más reciente concuerda en que la inclusión de estrategias que facilitan la comunicación entre la PeCP y su entorno posee tres objetivos universales, siendo responsabilidad del/la fonoaudiólogo/a del EdS favorecer su logro (Chahda et al., 2017; Eckman \& Roe, 2005; Pollens, 2012). Estos objetivos son:

(1) Mantener la cercanía emocional y social de la PeCP con sus familiares, cuidadores y otras personas significativas.

(2) Optimizar la habilidad de la PeCP para expresar sus necesidades, preocupaciones y preferencias relacionadas con su cuidado cotidiano y las decisiones al final de la vida.

(3) Preservar en la PeCP la habilidad para expresar sus estados emocionales, como cualquier otra preocupación referente a la muerte. Esto incluye alcanzar una sensación de cercanía espiritual y emocional previa a la muerte.

Tomando en consideración los objetivos previamente descritos, la intervención fonoaudiológica de la comunicación de PeCP toma como propósito mantener (durante el mayor tiempo posible), el control de la persona sobre sus cuidados y planes de fin de vida, permitiendo además interacciones significativas con su familia, amigos y cercanos (Kelly et al., 2016).

\section{Estructura general del plan de intervención comunicativa}

La evidencia actual establece la determinación de aquellas estrategias que permiten incrementar su efectividad comunicativa como pilar de todo plan de intervención comunicativa en PeCP (Chahda et al., 2017; Pollens, 2012), escogiendo aquellas que permiten disminuir de mejor manera las brechas que se generan en la interacción de la PeCP con sus interlocutores. Con fines didácticos, y sin desconocer que son muy variadas y dependientes de las características de la PeCP y su entorno, dichas estrategias pueden ser agrupadas en dos grandes categorías para facilitar su conceptualización (Chahda et al., 2017; Kelly et al., 2016):

a. Entrenamiento de los interlocutores en estrategias de andamiaje conversacional, permitiéndoles comprender con mayor claridad las intenciones comunicativas enunciadas por la PeCP, y.

b. Implementación de sistemas de comunicación aumentativa o alternativa, maximizando las capacidades comunicativas de la PeCP, disminuyendo las interferencias existentes particularmente en su expresión.
Tal como ha sido la tónica para otras intervenciones ya descritas, la evidencia plantea como característica fundamental de la intervención comunicativa en PeCP -a diferencia de aquella entregada a personas con condiciones no progresivas- una profunda flexibilidad para permitir su adaptación a los frecuentes cambios comunicativos y cognitivos que sobrelleva la persona a medida que su estado de salud declina (Kelly et al., 2016; Salt \& Robertson, 1998). Estas características otorgan a la intervención fonoaudiológica una condición de soporte y acompañamiento, la cual se prolonga hasta el final de la vida de la PeCP, con una continuidad que muchas veces es descrita como intermitente, como respuesta a los cambios que van ocurriendo en la persona (Chahda et al., 2017; Pollens, 2012).

\section{El rol de la comunicación en el alivio del sufrimiento}

Sobrellevar una enfermedad terminal genera un cambio en la personalidad de la persona afectada, viéndose forzada a moverse desde la independencia a la dependencia, desde la salud a la enfermedad, desde tener el control al no tenerlo (Goldberg, 2007). Dicha condición genera una profunda modificación en su conceptualización del mundo, como también del lugar que ocupa en este. Realidad que es puesta de manifiesto especialmente cuando desean comunicar su sufrimiento psicológico, instancias en que el/la fonoaudiólogo/a toma un importante rol apoyando, como colaborador/a y/o facilitador/a, en el alivio de este (Goldberg, 2007). En este contexto, la literatura refuerza la idea de posicionar a la comunicación no solo como una herramienta que permite el intercambio de información, sino como una forma que favorece la contención y el bienestar psicoemocional de la persona. En este contexto, Goldberg (2007) plantea un esquema de abordaje desde la comunicación para aquellas áreas más frecuentemente vinculadas al sufrimiento de la PeCP. Este esquema resulta ser uno de los más utilizado como lineamiento base por gran parte de las guías o recomendaciones clínicas existentes a la fecha para fonoaudiólogo/as que se desempeñan en cuidados paliativos, especialmente aquellas que declaran una orientación centrada en la humanización de los cuidados de fin de vida. A continuación, se revisan los elementos centrales de este esquema, en los cuales se establecen las orientaciones para el uso de la comunicación como herramienta terapéutica al servicio de PeCP y sus personas significativas.

\section{Comunicación para el ajuste en el cambio}

De acuerdo con Goldberg (2007) la persona que se acerca al final de su vida transita por un profundo cambio, volviéndose su mundo algo completamente inestable, en donde la persona que era hasta antes de la enfermedad desaparece progresivamente, 
transformándolo en alguien que se encuentra en constante transformación e impredictibilidad. En este contexto, el autor pone de manifiesto que la comunicación ha de alcanzar niveles de flexibilidad suficientes para dar cuenta de los cambios que ocurren en el proceso. Dicha cualidad de la comunicación se transforma, por ende, en uno de los ejes principales de la intervención fonoaudiológica, siendo imprescindible la implementación de las adecuaciones y compensaciones suficientes para que la $\mathrm{PeCP}$ reciba la contención $\mathrm{y}$ acompañamiento que necesita de sus personas significativas.

\section{Comunicación para entregar y recibir gratitud}

Entregar y recibir gratitud se transforma en un acto de alta importancia para el bienestar psico-emocional en el contexto de los cuidados paliativos (Goldberg, 2007). Permite a la PeCP cerrar ciclos y procesos con aquellas personas más significativas, además de fortalecer los vínculos que emergen en las dinámicas con su(s) cuidador(es). Si bien, para muchos individuos esto ocurre de manera natural y espontánea, para un número no menor resulta un proceso difícil de abordar (Emmons \& McCullough, 2004; Goldberg, 2007). En ambos casos, se plantea como recomendación el ofrecer un acompañamiento fonoaudiológico a la $\mathrm{PeCP}$ y sus personas significativas, que permita asegurar una comunicación eficiente que funcione como andamiaje para este tipo de interacciones.

\section{Comunicación y el arrepentimiento}

No es poco frecuente que quienes se aproximan a la muerte tengan arrepentimiento sobre cosas que han hecho y/o por aquellas que desearían haber realizado. En este contexto, la comunicación debe facilitar la entrega y recepción de disculpas, además de una positiva elaboración sobre aquellas metas no alcanzadas (Goldberg, 1997, 2007). Ante situaciones como estas, gran parte de lo/as profesionales tienden a responder minimizando dichas preocupaciones, o bien, cambiando la conversación hacia otros temas menos dolorosos, sin embargo, al no ser resultas, pueden transformarse en barreras para una muerte pacífica de la $\mathrm{PeCP}$ (Goldberg, 2006, 2007). En este contexto, se planta como rol del/la fonoaudiólogo/a propiciar un adecuado acompañamiento que favorezca -bajo de las condiciones que supone el contexto individual de cada PeCP- la optimización de la comunicación para resolver dichos temas pendientes.

\section{Comunicación y la necesidad de simplificar}

Cuando la muerte se acerca, la comunicación sufre un proceso de simplificación que frecuentemente no es comprendido por el entorno, especialmente la familia. La comunicación cotidiana, principalmente verbal, es reemplazada paulatinamente por otras formas. Toman mayor relevancia los gestos, la música o simplemente un abrazo; por sobre conversaciones extendidas y/o elaboradas (Goldberg, 1997, 2007). Por tanto, el autor plantea como responsabilidad del/la fonoaudiólogo/a el desarrollar estrategias que potencien este tipo de comunicación, considerando además un proceso previo de psicoeducación particularmente a la familia, personas significativas y EdS- con el fin de favorecer una adecuada y positiva resignificación de otras formas de comunicación.

\section{Recomendaciones para el acompañamiento de la persona que muere}

Sin lugar a duda uno de los elementos más complejos para el el/la fonoaudiólogo/a que se desempeña en cuidados paliativos resulta el enfrentar la muerte de manera cercana. Por ello, no es de extrañar que la comunicación (e incluso el vínculo) se vea interferida por variables que no son solo dependientes de la condición de la PeCP, sino que también por aspectos que le son propios a la persona tras el/la profesional (Goldberg, 2006).

$\mathrm{Al}$ respecto, Goldberg (2006), uno de los pioneros en abordar la comunicación en cuidados paliativos desde una perspectiva fonoaudiológica y humanizada, plantea una serie de recomendaciones mínimas para el acompañamiento de personas en cuidados de fin de vida (ver Tabla 4). Se espera que estas sean facilitadas por el/la fonoaudiólogo, permitiendo así su consideración por la familia, personas significativas y el resto del EdS.

Tabla 4. Recomendaciones para el acompañamiento de la persona que muere, traducido y adaptado por los autores a partir de Goldberg (2006).

Los "SÍ" $\quad$ Tratar a la persona que muere como alguien que se respeta profundamente.
Al hablar con la persona que está muriendo, hacerlo desde cerca permitiendo la interacción cara a cara.
Tocar a la persona, si esta se siente confortable con ello.
Comprender que el duelo no comienza con la muerte de la persona.
Aceptar amablemente la gratitud que la persona que muere pueda demostrar.
Decir adiós cada vez que se deja la habitación.
Conocer los signos físicos de la fase activa de la muerte.


Los "NO" Pretender que la persona está mejor cuando es obvio que no lo está.

Evitar hablar de la muerte, cuando la persona que muere si lo desea.

Hablar de la muerte, cuando la persona que muere no ha tocado el tema.

Tener temor por decir "no sé".

Aconsejar a la persona que muere sobre cosas que debería (o no) hacer.

Asumir que la muerte es igual para todos.

Asumir que la persona que muere puede hablar honestamente con otras personas, además de ti.

Tener miedo de sentirse afectado emocionalmente.

Asumir que veras a la persona la siguiente semana (o día).

Tener miedo de la muerte y de vivir un duelo por la persona.

\section{DISCUSIÓN}

Este artículo provee de información relevante, en base a una revisión de la literatura, sobre la práctica fonoaudiológica en adultos que requieren de cuidados paliativos. Para ello exploramos los aspectos que sirven como consideraciones transversales a toda intervención, permitiendo recabar información actualizada sobre los roles, áreas de acción y responsabilidades del/a profesional fonoaudiólogo/a. También se han descrito aquellos lineamientos básicos, que permiten un adecuado manejo de los cambios en la comunicación y alimentación de $\mathrm{PeCP}$, con el propósito de maximizar su bienestar psico-emocional -y el de su familia y personas significativas-, disminuir su dolor y sufrimiento, e incrementar su confort.

Se reconoce así que la práctica profesional en cuidados paliativos demanda de habilidades y competencias complejas., las cuales deben conjugarse a un proceder basado en la evidencia, principios éticos y un modelo de trabajo interdisciplinario (Kelly et al., 2016). A continuación, discutiremos sobre los alcances de los elementos tratados, planteados a modo de desafíos para consolidar la práctica fonoaudiológica en equipos de cuidados paliativos de nuestro país.

\section{Primer desafío: Cambio de paradigma}

Al observar la inclusión de los cuidados paliativos en la atención de salud de Chile, y las limitantes que emergen en su implementación, resulta significativo considerar el paradigma a la base de los servicios de salud. Los cuales históricamente han estado relacionados con la idea de curar, mejorar funciones y restaurar déficit; todas ideas provenientes del modelo biomédico (Baeta, 2015), el cual se aleja de los requerimientos vinculados a los cuidados paliativos y de fin de vida. Es por ello, que mucho/as profesionales de la salud -e incluso muchas de las PeCP y sus respectivas familias o personas significativas-consideran aún el acompañamiento en el proceso de muerte como una renuncia a la vida (García-Caro et al., 2010).
Es necesario, por tanto, relevar el modelo biopsicosocial como un paradigma más comprensivo de la situación global de la persona, que explora más allá de lo biológico y considerada aspectos emocionales, sociales, espirituales, entre otros. Bajo este paradigma se busca comprender las complejidades propias de cada persona y su entorno, siendo particularmente relevante al enfrentar situaciones de salud complejas, multifactoriales y en procesos que implican la muerte. Bajo lo expuesto, una real aplicación de los cuidados paliativos implica una evolución desde los tradicionales modelos de "rehabilitación", hacia uno que comprenda la salud y bienestar de las personas en un constructo que considere tanto la calidad de vida, como el derecho a morir dignamente. Este punto resulta un elemento central en la reflexión que deben realizar lo/as profesionales de la salud a su práctica clínica, incluido/as lo/as fonoaudiólogo/as, permitiéndoles poner en práctica el paradigma biopsicosocial al tiempo que se alejan del modelo rehabilitador, para acercarse a un modelo basado en los cuidados. Dicha aproximación permite resaltar lo significativo de comenzar con los cuidados paliativos cuando la persona lo necesita, entendiendo que no deben ser postergados o relegados como una última opción.

\section{Segundo desafío: Definir los roles de fonoaudiólogo/as en cuidados paliativos}

El valor que poseen lo/as fonoaudiólogo/as especialistas en cuidados paliativos parece no estar aún completamente reconocido por quienes son fonoaudiólogo/as generales, tampoco por otro/as profesionales que aún no han tenido la oportunidad de trabajar con fonoaudiólogo/as dedicados a cuidados paliativos (Kelly et al., 2016; Toner \& Shadden, 2012; Vescovich, 2015); siendo necesaria una mayor educación a la población en general, el equipo de salud y gestores de políticas públicas, respecto a los beneficios de su temprana inclusión en los cuidados paliativos.

Lo anterior, permitiría disminuir la recurrente percepción sobre la fonoaudiología como un servicio extra o adicional a los cuidados paliativos, condición que explicaría por qué su inclusión ocurre 
frecuentemente después del momento considerado como ideal para favorecer cambios en la calidad de vida de la persona (Kelly et al., 2016; Roe \& Leslie, 2010). Emerge así, como desafío necesario, la generación de un consenso entre lo/as fonoaudiólogo/as con experiencia en cuidados paliativos, que permita establecer un lenguaje y lineamientos que sean comunes y propios del área; alejándose del esquema rehabilitador y otorgando un mayor valor al importante rol que cumplen en los cuidados paliativos.

\section{Tercer desafío: Preparación de futuro/as profesionales}

En un contexto donde la población chilena progresivamente envejece (Albala, 2020), la probabilidad de que un/a fonoaudiólogo/a preste servicios a una $\mathrm{PeCP}$ es cada vez más alta. Esto se contrapone con la formación que la mayoría de las universidades otorga durante la formación de pregrado, donde el desarrollo intencionado de competencias orientadas a lidiar con el impacto de trabajar con personas que mueren es limitado. Al respecto, se ha reconocido la efectividad que poseen la inclusión de espacios de reflexión y de lectura crítica guiados por profesionales con experiencia en el área; permitiendo incluir el razonamiento paliativo a lo largo de la formación de pregrado (Kelly et al., 2016; Mathisen et al., 2011). Idealmente, dichas instancias deben ser realizadas utilizando metodologías que integren a los estudiantes de fonoaudiología con estudiantes de diferentes disciplinas (Mathisen et al., 2011); como trabajo social, enfermería y/o medicina, fomentando tempranamente el trabajo interdisciplinario basado en consensos. En este contexto, surge un nuevo desafío para quienes ocupan el rol de formadores, a quienes se les invita a desarrollar e implementar planes curriculares que incluyan los principios y competencias vinculadas a los cuidados paliativos.

\section{Cuarto desafío: Competencias para trabajar con personas que mueren}

No es poco frecuente ver emerger la errada creencia de que todo/a profesional con el título de fonoaudiólogo/a puede desempeñarse satisfactoriamente en equipos de cuidados paliativos, desconociendo con ello las particularidades a las cuales debe hacer frente; no solo en lo técnico o profesional, sino que muchas veces en lo personal. Trabajar en cuidados paliativos implica un profundo desafío en donde: (a) el planteamiento de objetivos, (b) las incertezas, y (c) la definición de hasta donde llegar en los cuidados, se transforman en un complejo escenario, especialmente para quienes acostumbran a desempeñarse bajo un modelo rehabilitador (Kelly et al., 2016; Roe \& Leslie, 2010). El desenvolverse en un área donde cotidianamente se lidia con la muerte y personas muriendo contribuye al estrés y burnout de lo/as profesionales, pudiendo derivar en una baja en la calidad de los servicios y atención entregada (Kelly et al., 2016). Del mismo modo, el estar cotidianamente expuesto/a al sufrimiento puede derivar en una fatiga de la compasión, comprometiendo seriamente la empatía del/la profesional (Kelly et al., 2016; Toner \& Shadden, 2012).

Desempeñarse en cuidados paliativos es un trabajo complejo, siendo necesario contar con las competencias suficientes para afrontar de manera adecuada el trabajar con personas que mueren. Esto releva la necesidad de contar con una adecuada preparación, la cual es específica a los cuidados paliativos, y da cuenta de la necesidad de propiciar adecuados procesos de supervisión clínica, debriefing, soporte entre pares, mentoría y educación (Kelly et al., 2016). En la actualidad, son escasas las oportunidades para abordar estas necesidades, existiendo programas de educación continua en cuidados paliativos -generalmente destinados a médico/as y enfermero/as- que se orientan esencialmente a lo técnico, en desmedro de aquellas competencias orientadas a afrontar la muerte.

\section{Quinto desafío: Creación de guías de práctica fonoaudiológica en cuidados paliativos}

Chile no es ajeno a una condición que es generalizada en la mayoría de los países, la falta de orientaciones y guías clínicas que permitan a lo/as fonoaudiólogo/as tener un marco sobre el cual desempeñarse en cuidados paliativos. Esto ha derivado en que la transferencia del conocimiento y recomendaciones clínicas generalmente provengan de profesionales con mayor experiencia. Profesionales que generalmente no son fonoaudiólogo/as, sino enfermero/as, quienes son reconocido/as frecuentemente como un importante soporte hasta que lo/as fonoaudiólogo/as logran mayor confianza en su proceder (Chahda et al., 2020).

Se posiciona entonces como nuevo desafío para lo/as profesionales del país el generar documentos que funcionen como guía específica para el actuar de lo/as fonoaudiólogo/as en cuidados paliativos. Orientaciones que, dada las características propias del quehacer en cuidados paliativos, deben estar estructuradas como una guía amplia para el proceder fonoaudiológico, más que un esquema paso a paso de acciones a realizar (Chahda et al., 2020).

\section{Sexto desafío: Modificaciones en las políticas de cuidados paliativos}

Actualmente, el servicio de salud chileno cuenta con un único programa orientado específicamente a los cuidados paliativos, el 
cual corresponde al de "Alivio del Dolor por Cáncer y Cuidados Paliativos", el cual surge el año 1994 como parte del Plan Nacional de Cáncer, incorporando como pilar el modelo de continuidad de los cuidados, propuesto por el Programa de Lucha contra el Cáncer de la OMS (Ministerio de Salud [MINSAL], 2011). Sin embargo, no es hasta el año 2011 que este programa es incluido dentro de las Garantías Explícitas de Salud (GES) para la población chilena. Lamentablemente, este programa no considera a el/la fonoaudiólogo/a como parte del equipo de acompañamiento, a pesar de la evidencia que da cuenta de cómo los tratamientos, y la propia evolución del cáncer, impactan significativamente en su alimentación (Sarría et al., 2007).

A partir de lo expuestos emergen dos desafíos que deben ser proyectados tanto por profesionales fonoaudiólogo/as, como por el resto de lo/as profesionales que son parte de los cuidados paliativos. Como condición obvia, se hace necesaria la inclusión de los servicios fonoaudiológicos en programas como este, permitiendo favorecer la calidad de vida de quienes lo requieren. En segundo término, resulta necesario aunar esfuerzos para favorecer una visión más amplia de los cuidados paliativos, fomentando la generación de políticas de salud que permitan acoger a personas que los requieran, ya sea producto de una enfermedad degenerativa, o no. Al respecto, la generación de evidencia que, de cuenta de sus beneficios a nivel nacional, se transforma en uno de los medios más apropiados para ello.

\section{CONCLUSIONES}

Considerando el aún incipiente desarrollo de la práctica fonoaudiológica en cuidados paliativos, este documento entrega relevantes orientaciones para la práctica fonoaudiológica bajo un modelo de servicio orientado al cuidado y bienestar. Hemos explorado roles, áreas de acción, responsabilidades y marcos teóricos que permiten describir las particularidades que debe poseer el o la profesional que se desempeña en cuidados paliativos, dando cuenta de competencias que le son propias y distintivas de otras áreas de especialización fonoaudiológica.

En particular, los resultados de esta revisión permiten establecer una serie de desafíos para los clínicos que esperamos sean abordados con una clara orientación hacia la generación de cambios en nuestra práctica clínica. Cambios que se soportan especialmente en el reconocimiento de la necesidad de alejarse de modelos tradicionales de intervención, para empaparse de aquellos que buscan ciertamente transformar a este/a profesional en un adecuado compañero en el tránsito hacia un buen morir. Esperamos que este documento favorezca una mayor discusión sobre el tema entre lo/as profesionales de la salud, al tiempo que sea útil como guía para orientar a quienes recién se incorporan (o desean hacerlo) en el ámbito de los cuidados paliativos. Finalmente, invitamos a quienes ya cuentan con práctica en el área, a compartir sus experiencias y fomentar el desarrollo de nuestro quehacer en beneficio de quienes lo necesiten.

\section{REFERENCIAS}

Aguirre Bravo, A. N., \& Sampallo Pedroza, R. M. (2015). Fonoaudiología en los Cuidados Paliativos. Revista de la Facultad de Medicina, 63(2), 289-300. https://doi.org/10.15446/revfacmed.v63n2.48539

Albala, C. (2020). El envejecimiento de la población chilena y los desafíos para la salud y el bienestar de las personas mayores. Revista Médica Clínica Las Condes, 3l(1), 7-12. https://doi.org/10.1016/j.rmclc.2019.12.001

Alvarez-Fernández, B., García-Ordoñez, M. A., Martínez-Manzanares, C., \& Gómez-Huelgas, R. (2005). Survival of a cohort of elderly patients with advanced dementia: Nasogastric tube feeding as a risk factor for mortality. International Journal of Geriatric Psychiatry, 20(4), 363-370. https://doi.org/10.1002/gps.1299

Arinzon, Z., Peisakh, A., \& Berner, Y. N. (2008). Evaluation of the Benefits of Enteral Nutrition in Long-Term Care Elderly Patients. Journal of the American Medical Directors Association, 9(9), 657-662. https://doi.org/10.1016/j.jamda.2008.06.002

Arksey, H., \& O'Malley, L. (2005). Scoping studies: Towards a methodological framework. International Journal of Social Research Methodology, 8(1), 19-32. https://doi.org/10.1080/1364557032000119616

Baeta, M. (2015). Cultura y modelo biomédico: Reflexiones en el proceso de salud enfermedad. Comunidad y Salud, 13(2), 81-84. http://ve.scielo.org/scielo.php?script=sci_abstract\&pid=S1690$32932015000200011 \& \operatorname{lng}=\mathrm{es} \& n r m=\mathrm{iso} \overline{\mathrm{t}} \operatorname{lng}=\mathrm{es}$

Berkman, C., Ahronheim, J. C., \& Vitale, C. A. (2019). Speech-Language Pathologists' Views About Aspiration Risk and Comfort Feeding in Advanced Dementia. American Journal of Hospice and Palliative Medicine ${ }^{\circledR}, 36(11)$, 993998. https://doi.org/10.1177/1049909119849003

Burlá, C., \& Py, L. (2014). Palliative care: Science and protection at the end of life. Cadernos de Saúde Pública, 30, 1139-1141. https://doi.org/10.1590/0102311XPE020614

Carter, A. N. (2020). To What Extent Does Clinically Assisted Nutrition and Hydration Have a Role in the Care of Dying People? Journal of Palliative Care, 35(4), 209-216. https://doi.org/10.1177/0825859720907426

Chahda, L., Carey, L. B., Mathisen, B. A., \& Threats, T. (2020). Speech-language pathologists and adult palliative care in Australia. International Journal of Speech$\begin{array}{lll}\text { Language } & \text { Pathology, } & \text { 23(1), }\end{array}$ https://doi.org/10.1080/17549507.2020.1730966

Chahda, L., Mathisen, B. A., \& Carey, L. B. (2017). The role of speech-language pathologists in adult palliative care. International Journal of Speech-Language Pathology, 19(1), 58-68. https://doi.org/10.1080/17549507.2016.1241301

Cintra, M. T. G., de Rezende, N. A., de Moraes, E. N., Cunha, L. C. M., \& da Gama Torres, H. O. (2014). A comparison of survival, pneumonia, and hospitalization in patients with advanced dementia and dysphagia receiving either oral or enteral nutrition. The Journal of Nutrition, Health \& Aging, 18(10), 894 


\section{9. https://doi.org/10.1007/s12603-014-0487-3}

Eckman, S., \& Roe, J. (2005). Speech and language therapists in palliative care: What do we have to offer? International Journal of Palliative Nursing, 11(4), 179181. https://doi.org/10.12968/ijpn.2005.11.4.28783

Emmons, R. A., \& McCullough, M. E. (Eds.). (2004). The Psychology of Gratitude. Oxford University Press.

Evans, L., \& Best, C. (2015). Managing malnutrition in patients with dementia. Nursing Standard, 29(28), 50-57. https://doi.org/10.7748/ns.29.28.50.e9752

Finucane, T. E., Christmas, C., \& Travis, K. (1999). Tube feeding in patients with advanced dementia: A review of the evidence. JAMA, 282(14), 1365-1370. https://doi.org/10.1001/jama.282.14.1365

Fong, R., Tsai, C.-F., Wong, W. H.-S., Yiu, O.-Y., \& Luk, J. K. H. (2020). Speech therapy in palliative care and comfort feeding: Current practice and way ahead. Asian Journal of Gerontology and Geriatrics, 14(2), 61-68. https://doi.org/10.12809/ajgg-2018-330-oa

García-Caro, M. P., Cruz-Quintana, F., Río-Valle, J. S., Muñoz-Vinuesa, A., Montoya-Juárez, R., Prados-Peña, D., Pappous, A., \& Botella-López, M. C. (2010). Influencia de las emociones en el juicio clínico de los profesionales de la salud a propósito del diagnóstico de enfermedad terminal. International Journal of Clinical and Health Psychology, 10(1), 57-73. https://www.redalyc.org/articulo.oa?id=33712017004

Gillick, M. R. (2000). Rethinking the Role of Tube Feeding in Patients with Advanced Dementia. New England Journal of Medicine, 342(3), 206-210. https://doi.org/10.1056/NEJM200001203420312

Goldberg, S. (1997). Clinical Skills For Speech-Language Pathologists: Practical Applications. Singular Pub. Group.

Goldberg, S. (2006). Shedding Your Fears: Bedside Etiquette for Dying Patients. Topics in Stroke Rehabilitation, 13(1), 63-67. https://doi.org/10.1310/F9XRU2AT-CACK-0PV3

Goldberg, S. (2007). There's an elephant in the room: Issues in death and dying. En A. Holland \& L. Nelson (Eds.), Counseling in Communication Disorders: A Wellness Perspective (pp. 261-280). Singular Pub. Group.

Gomes, G. F., Pisani, J. C., Macedo, E. D., \& Campos, A. C. (2003). The nasogastric feeding tube as a risk factor for aspiration and aspiration pneumonia. Current Opinion in Clinical Nutrition \& Metabolic Care, 6(3), 327-333. https://doi.org/10.1097/01.mco.0000068970.34812.8b

Holland, A., \& Nelson, L. (Eds.). (2007). There's an elephant in the room: Issues in death and dying. Singular Pub. Group.

Ijaopo, E. O., \& Ijaopo, R. O. (2019). Tube Feeding in Individuals with Advanced Dementia: A Review of Its Burdens and Perceived Benefits. Journal of Aging Research, 1-16. https://doi.org/10.1155/2019/7272067

Instituto Nacional de Estadísticas [INE]. (2018). Estimaciones y proyecciones de la población de Chile 1992-2050. Total país. https://www.ine.cl/docs/defaultsource/proyecciones-de-poblacion/publicaciones-y-anuarios/base-

2017/ine_estimaciones-y-proyecciones-de-poblaci\%C3\%B3n-1992-2050_base-

2017 _s\%C3\%ADntesis.pdf?sfvrsn=c623983e_6

Kelly, K., Cumming, S., Corry, A., Gilsenan, K., Tamone, C., Vella, K., \& Bogaardt, H. (2016). The role of speech-language pathologists in palliative care: Where are we now? A review of the literature. Progress in Palliative Care, 24(6), 315-323. https://doi.org/10.1080/09699260.2016.1141745

Langmore, S. E., Grillone, G., Elackattu, A., \& Walsh, M. (2009). Disorders of
Swallowing: Palliative Care. Otolaryngologic Clinics of North America, 42(1), 87-105. https://doi.org/10.1016/j.otc.2008.09.005

Levy, A., Dominguez-Gasson, L., Brown, E., \& Frederick, C. (2004). Managing Dysphagia in the Adult Approaching End of Life. The ASHA Leader, 9(3), 1-17. https://doi.org/10.1044/leader.FTR1.09132004.1

Luk, J. K., \& Chen, D. K. (2014). Preventing aspiration pneumonia in older people: Do we have the "know-how"? HKMJ, 20, 421-427. https://www.hkmj.org/abstracts/v20n5/421.htm

Mamun, K., \& Lim, J. (2005). Role of nasogastric tube in preventing aspiration pneumonia in patients with dysphagia. Singapore Medical Journal, 46(11), 627 631.

https://pubmed.ncbi.nlm.nih.gov/16228094/\#: :text=Nasogastric\%20tube\%2Dfe d\%20patients\%20were,who\%20were\%20on\%20oral\%20feeding.

Mathisen, B., Yates, P., \& Crofts, P. (2011). Palliative care curriculum for speechlanguage pathology students. International Journal of Language \&

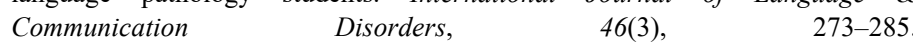
https://doi.org/10.3109/13682822.2010.495739

Ministerio de Salud [MINSAL]. (2011). Guía Clínica Alivio del Dolor por Cáncer Avanzado $y \quad$ Cuidados Paliativos. http://www.repositoriodigital.minsal.cl/bitstream/handle/2015/253/Alivio-delDolor-por-C\%c3\%a1ncer-y-Cuidados-Paliativos.pdf?sequence=1\&isAllowed=y

Ministerio de Sanidad, Política Social e Igualdad. (2014). Estrategia en Cuidados Paliativos del Sistema Nacional de Salud. Actualización 2010-2014. Ministerio de Sanidad, Política Social e Igualdad Centro de Publicaciones. https://www.mscbs.gob.es/organizacion/sns/planCalidadSNS/docs/paliativos/cui dadospaliativos.pdf

Moreira, M. J. da S., Guimarães, M. F., Lopes, L., \& Moreti, F. (2020). Contribuições da Fonoaudiologia nos cuidados paliativos e no fim da vida. CoDAS, 32. https://doi.org/10.1590/2317-1782/20202019202

Nakanishi, M., \& Hattori, K. (2014). Percutaneous endoscopic gastrostomy (PEG) tubes are placed in elderly adults in Japan with advanced dementia regardless of expectation of improvement in quality of life. The Journal of Nutrition, Health \& Aging, 18(5), 503-509. https://doi.org/10.1007/s12603-014-0011-9

Naruishi, K., \& Nishikawa, Y. (2018). Swallowing impairment is a significant factor for predicting life prognosis of elderly at the end of life. Aging Clinical and Experimental Research, 30(1), 77-80. https://doi.org/10.1007/s40520-017-07561

National Institute for Clinical Excellence [NICE]. (2004). Guidance on cancer services: Improving supportive and palliative care for adults with adults the manual. National Institute for Clinical Excellence. https://discover.libraryhub.jisc.ac.uk/search?ti=Guidance $\% 20$ on $\% 20$ Cancer $\% 20$ Services $\% 3$ A $\% 20$ Improving\%20Supportive $\% 20$ and $\% 20$ Palliative $\% 20$ care $\% 20 \mathrm{f}$ or $\% 20$ Adults\&rn $=5$

Organización Mundial de la Salud [OMS]. (2020, agosto 20). Cuidados paliativos. https://www.who.int/es/news-room/fact-sheets/detail/palliative-care

Palecek, E. J., Teno, J. M., Casarett, D. J., Hanson, L. C., Rhodes, R. L., \& Mitchell, S. L. (2010). Comfort Feeding Only: A Proposal to Bring Clarity to Decision-Making Regarding Difficulty with Eating for Persons with Advanced Dementia. Journal of the American Geriatrics Society, 58(3), 580-584. https://doi.org/10.1111/j.1532-5415.2010.02740.x

Pollens, R. D. (2012). Integrating Speech-Language Pathology Services in Palliative End-of-Life Care. Topics in Language Disorders, 32(2), 137-148. https://doi.org/10.1097/TLD.0b013e3182543533 
Portales, M. B. (2015). A survey about the criteria for the indication of a gastrostomy in patients with advanced dementia. Revista Médica de Chile, 143(11), 1405-1410. https://doi.org/10.4067/S0034-98872015001100005

Prelock, P. A., Melvin, C., Lemieux, N., Melekis, K., Velleman, S., \& Favro, M. A. (2017). One Team-Patient, Family, and Health Care Providers: An Interprofessional Education Activity Providing Collaborative and Palliative Care. Seminars in Speech and Language, 38(05), 350-359. https://doi.org/10.1055/s0037-1607071

Prince, M. J., Wu, F., Guo, Y., Robledo, L. M. G., O’Donnell, M., Sullivan, R., \& Yusuf, S. (2015). The burden of disease in older people and implications for health policy and practice. The Lancet, 385(9967), 549-562. https://doi.org/10.1016/S0140-6736(14)61347-7

Rochford, A. (2021). Ethics of providing clinically assisted nutrition and hydration: Current issues. Frontline Gastroenterology, 12(2), 128-132. https://doi.org/10.1136/flgastro-2019-101230

Roe, J. W. G., \& Leslie, P. (2010). Beginning of the end? Ending the therapeutic relationship in palliative care. International Journal of Speech-Language Pathology, 12(4), 304-308. https://doi.org/10.3109/17549507.2010.485330

Royal College of Physicians and British Society of Gastroenterology. (2010). Oral feeding difficulties and dilemmas: A guide to practical care, particularly towards the end of life. https://shop.rcplondon.ac.uk/products/oral-feeding-difficultiesand-dilemmas-a-guide-to-practical-care-particularly-towards-the-end-of-life

Salt, N., \& Robertson, S. J. (1998). A Hidden Client Group? Communication Impairment in Hospice Patients. International Journal of Language \& Communication $\quad$ Disorders, $\quad$ 33(S1), 101. https://doi.org/10.3109/13682829809179404

Sarría, P., Trobat, D., \& Epprecht, P. (2007). Trastornos de la deglución postradioterapia. En G. Jaume Bauza \& M. Tomas Barberan (Eds.), Manejo de la disfagia y aspiración (pp. 93-96). Ergon.

Teramoto, S., Ishii, T., Yamamoto, H., Yamaguchi, Y., \& Ouchi, Y. (2006). Nasogastric tube feeding is a cause of aspiration pneumonia in ventilated patients. European Respiratory Journal, 27(2), 436-437. https://doi.org/10.1183/09031936.06.00113405

Toner, M. A., \& Shadden, B. B. (2012). End of Life: An Overview. Topics in Language Disorders, 32(2), 111-118. https://doi.org/10.1097/TLD.0b013e31825484e0

Vescovich, M. (2015). Holding On... and Letting Go. The ASHA Leader, 20(2), 48-53. https://doi.org/10.1044/leader.FTR2.20022015.48 